\title{
Digital interference microscopy and density reconstruction of picosecond infrared laser desorption at the water-air interface
}

Frederik Busse, Sebastian Kruber, Wesley D. Robertson, and R. J. Dwayne Miller

Citation: Journal of Applied Physics 124, 094701 (2018); doi: 10.1063/1.5030741

View online: https://doi.org/10.1063/1.5030741

View Table of Contents: http://aip.scitation.org/toc/jap/124/9

Published by the American Institute of Physics

\section{Articles you may be interested in}

Comparison of unit cell coupling for grating-gate and high electron mobility transistor array $\mathrm{THz}$ resonant absorbers

Journal of Applied Physics 124, 093101 (2018); 10.1063/1.5032102

Raman analysis of strain in p-type doped silicon nanostructures

Journal of Applied Physics 124, 095102 (2018); 10.1063/1.5045736

Incorporation of Si during vapor phase epitaxy of III-V compounds: Evidence of an enthalpy-entropy compensation effect

Journal of Applied Physics 124, 095303 (2018); 10.1063/1.5031106

Surface reaction kinetics in atomic layer deposition: An analytical model and experiments Journal of Applied Physics 124, 095302 (2018); 10.1063/1.5044456

Effect of rectangular grooves and checkerboard patterns on the electron emission yield Journal of Applied Physics 124, 095101 (2018); 10.1063/1.5028216

A study of irradiation effects in $\mathrm{TiO}_{2}$ using molecular dynamics simulation and complementary in situ transmission electron microscopy

Journal of Applied Physics 124, 095901 (2018); 10.1063/1.5045491

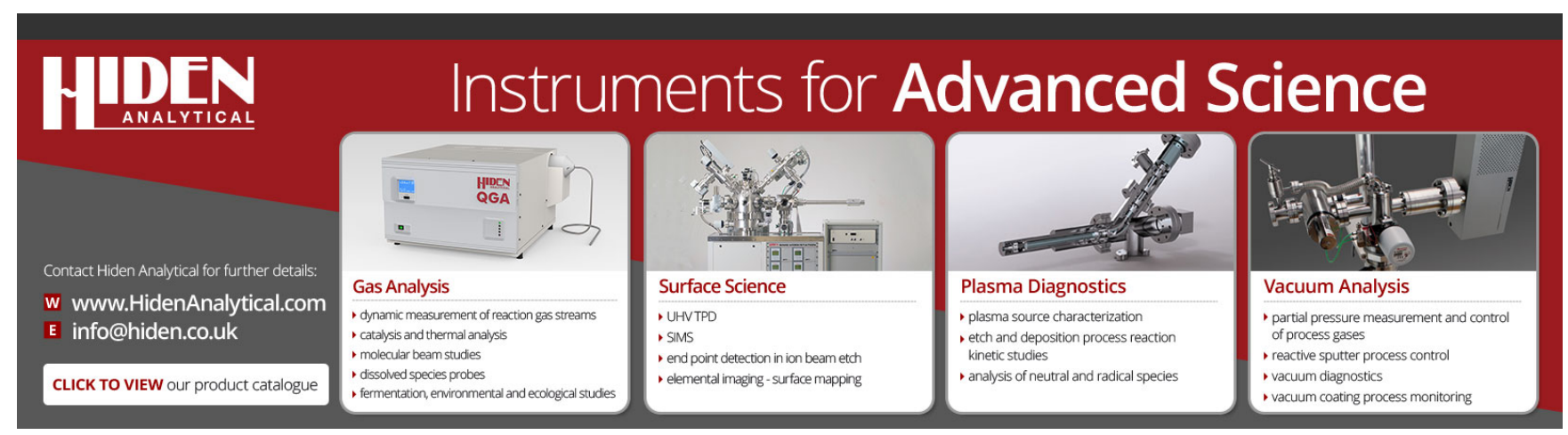




\title{
Digital interference microscopy and density reconstruction of picosecond infrared laser desorption at the water-air interface
}

\author{
Frederik Busse, ${ }^{1,2}$ Sebastian Kruber, ${ }^{1,2}$ Wesley D. Robertson, ${ }^{1}$ and R. J. Dwayne Miller ${ }^{1,3, a)}$ \\ ${ }^{1}$ Max Planck Institute for the Structure and Dynamics of Matter, Centre for Ultrafast Imaging, \\ Luruper Chaussee 149, 22761 Hamburg, Germany \\ ${ }^{2}$ Department of Physics, University of Hamburg, Jungiusstraße 9-11, 20355 Hamburg, Germany \\ ${ }^{3}$ Departments of Chemistry and Physics, University of Toronto, 80 St. George Street, Toronto, \\ Ontario M5S 3H6, Canada
}

(Received 25 March 2018; accepted 24 July 2018; published online 6 September 2018)

\begin{abstract}
Material ablation and evaporation using pulsed infrared lasers pose promising approaches for matrix-free laser desorption ionization and in laser surgery. For the best results, key parameters such as laser wavelength, pulse duration, and pulse energy need to be carefully adjusted to the application. We characterize the dynamics at the water-air interface induced by a 10 ps infrared laser tuned to the water absorption band at $3 \mu \mathrm{m}$, a parameter set facilitating stress confined desorption for typical absorption depths in biological samples and tissue. By driving the ablation faster than nucleation growth, cavitation induced sample damage during the ablation process can be mitigated. The resultant explosive ablation process leads to a shock front expansion and material ejection which we capture using off-axis digital interference microscopy, an interference technique particularly useful for detecting the phase shift caused by transparent objects. It is demonstrated that the method can yield local density information of the observed shock front with a single image acquisition as compared to the usually performed fit of the velocity extracted from several consecutive snapshots. We determine the ablation threshold to be $(0.5 \pm 0.2) \mathrm{J} \mathrm{cm}^{-2}$ and observe a significant distortion of the central parts of the primary shock wave above approximately $2.5 \mathrm{~J} \mathrm{~cm}^{-2}$. The differences in plume shape observed for higher fluences are reflected in an analysis based on shock wave theory, which shows a very fast initial expansion. Published by AIP Publishing.

https://doi.org/10.1063/1.5030741
\end{abstract}

\section{INTRODUCTION}

Laser ablation is heavily utilized in numerous surface analysis techniques for the immediate and spatially resolved extraction of materials and has also been successfully applied to a variety of non-analytical applications including pulsed laser deposition, laser machining, and laser surgery. Imaging studies yield information on the characteristics of the ablation plume which is valuable for optimizing the conditions to achieve, e.g., improved material collection in mass spectrometry, ${ }^{1}$ minimal tissue damage in laser surgery, ${ }^{2-4}$ and homogeneous film growth in pulsed laser deposition. 5,6 Beyond that, the spatio-temporal information gained by imaging the ablation plume can be used to form more refined models describing the fundamental processes, leading to ablation and ionization. $^{7-9}$

Matrix assisted laser desorption ionization (MALDI) mass spectrometry, one of the most successful analytical applications of laser ablation, has become a standard for the analysis of (bio-)organic compounds, in particular for protein sequencing and proteomic research. Typically, MALDI is performed using ultraviolet (UV) laser radiation, and soft ablation and ionization are achieved by co-crystallizing or spraying the sample with an organic matrix, which serves to absorb the incoming photons. Infrared (IR) laser desorption has gained

${ }^{a)}$ Electronic mail: dwayne.miller@mpsd.mpg.de substantial interest as it facilitates a softer mode of ablation than UV-MALDI in that it leads to less (metastable) ion fragmentation and photochemistry. ${ }^{10-13}$ Additionally, many materials absorb strongly in the IR and can thus be used as MALDI matrices. Ablation of many samples of interest is even possible without an external agent, i.e., matrix-free, resulting in a very versatile technique which can, for example, be combined with liquid-phase separation techniques. ${ }^{14,15}$ Water has received specific attention as its strong IR absorption allows for the analysis of many biological systems in their native environment. ${ }^{15-17}$ Favorable conditions for material removal and analyte desolvation and potentially ionization are achieved under thermal- and stress-confinement as described by desorption by impulsive vibrational excitation (DIVE) for IR excitation of water. ${ }^{18,19}$ In DIVE, high energy densities are achieved by resonant excitation of the water $\mathrm{O}-\mathrm{H}$ stretching mode at $2.94 \mu \mathrm{m}$ with an excitation pulse duration below the timescale of shock-wave-propagation through the ablation volume. At the same time, efficient energy redistribution into thermal modes is ensured by keeping the irradiance low enough to avoid nonlinear optical excitation of higher-lying molecular states. Picosecond infrared laser (PIRL) irradiation fulfilling these criteria has been shown to be well-suited for the extraction of large bio-molecules and even intact viruses for subsequent offline analysis, ${ }^{20-22}$ as an atmospheric online ion source in combination with electrospray ionization (LAESI), ${ }^{23}$ and has been suggested as a potential tool for sample-delivery in high-speed 
X-ray crystallography. ${ }^{24}$ Moreover, the absence of ionizing radiation and minimal deposition of thermal and acoustic energy into the non-ablated sample surface make DIVE a valuable tool for precise material removal in surgical applications. ${ }^{25-27}$ While pulse durations above the thermal diffusion time ( $\approx 1 \mu$ s for typical IR laser desorption) are known to burn tissue and to yield mass spectra with high fragmentation ratios, shorter pulse durations facilitate a more efficient energy deposition in which energy diffusion is avoided, thus allowing for lower laser fluences. ${ }^{28}$ Additionally, the resulting short-lived high pressure gradients lead to a fast adiabatic cooling during plume expansion, which reduce the molecules' internal energy. ${ }^{18}$ If the energy density for a phase explosion is reached, condensed matter is more efficiently ejected via spallation, creating a fine mist of sample material at low temperature. Both effects and the influence of stress confinement have been studied extensively using molecular dynamics (MD) simulations. ${ }^{19,29,30}$ However, only few fundamental studies of IR ablation under actual stress-confinement (pulse durations $<1 \mathrm{~ns}$ for water ablation, shorter for materials with higher absorption) have been performed so far and most for solid samples. ${ }^{31,32}$ For liquid samples, pulse durations between $10 \mathrm{~ns}$ and $100 \mathrm{~ns}$ usually show a two-stage material emission, starting with an evaporative process which is followed by a phase explosion. ${ }^{4,8}$ When utilizing pulse durations of only a few nanoseconds, the onset of stress-confinement becomes apparent. ${ }^{33,34}$ Explicit differences in the plume dynamics between DIVE (100 ps pulse duration) and merely thermally confined IR laser ablation ( $70 \mathrm{~ns}$ pulse duration) have recently been demonstrated in an imaging study of IR laser ablation of liquid water, ${ }^{18}$ in which the authors employed dark-field microscopy to ensure proper ablation conditions.

In order to not only investigate the condensed matter ejected during plume expansion, such as splinters and droplets, but also gain information about the shock-wave-propagation and the gas-phase portions of the plume, a time-resolved, high-contrast imaging method capable of detecting minute changes in the refractive index is needed. Emission spectroscopy has been applied to the characterization of ablation plumes under laser breakdown conditions where plasma generation is a dominant process. ${ }^{35-37}$ For lower pulse energies, which are commonly employed for soft ablation of biological samples, i.e., in MALDI, no strong fluorescent emission is present. The addition of fluorescent markers to the sample has been used to provide information on the differences in angular spread of the analyte and matrix molecules. ${ }^{38,39}$ Most commonly, shadowgraphy, dark-field microscopy, and Schlieren photography are used to image laser ablation plumes. These label-free methods show high contrast even for desorbed molecules in the gas-phase which have low opacity. ${ }^{40}$ However, they give rise to certain ambiguities in interpretation as the recorded brightness is a measure of the refractive index gradient mixed with contributions from scattering and possibly fluorescence. In contrast, holographic recording methods show contrast directly proportional to the object's optical path length and thus contain quantitative information about the three-dimensional refractive index distribution of the illuminated object. ${ }^{41}$ They are therefore often used in biomedical ${ }^{42}$ or solid-state applications ${ }^{43}$ where otherwise transparent objects need to be imaged with high precision and sensitivity. While other microscopy approaches often require temporally incoherent illumination to avoid artifacts (i.e., by employing a multimode fiber $^{40}$ ) holography relies on a coherent laser source and is thus ideally suited for nanosecond time resolved imaging. High optical contrast is achieved without the necessity of blocking portions of the laser beam, and when using a double exposure scheme,${ }^{4,45}$ the noisy background can easily be subtracted while maintaining the absolute refractive index information.

In an extension of digital holography, extended-focusimaging, recorded holograms can be digitally refocused to not only correct for a shifted or tilted focal plane but also create a stack of refocused amplitude images at different distances from the real focal plane of the microscope objective and to ultimately create a three-dimensional representation of the object in question. ${ }^{46-48}$ However, for successful applications of this technique, the object needs to be opaque or its refractive index distribution needs to be known so that the topology can be inferred from the initial phase map. For this reason, it has so far only been applied to nontransparent or simple transparent objects with a constant refractive index and not to complex refractive index distributions which are found for laser ablation plumes. Three-dimensional representations of holographically recorded objects have also been acquired using a tomographic approach. ${ }^{49}$ This, however, requires recording the object from different projection angles, which is challenging, given the turbulent nature and fast evolution of the hydrodynamics observed for laser ablation plumes. The high contrast for otherwise transparent objects makes holographic microscopy a method of choice for characterizing such plumes and has in particular been used to study the free electron density distribution of breakdown plasmas created during the ablation of solid targets. ${ }^{50-52}$ However, absolute density calculations in this case are difficult unless several wavelengths are used or the free electron density is large enough $\left(>2 \times 10^{19} \mathrm{~cm}^{-3}\right)$ that the neutral density contribution to the refractive index can be neglected..$^{53}$ However, under the assumption that the shock is entirely composed of (neutral) ambient air ${ }^{9}$ and that the plume is rotationally symmetric, the method can be used to compute a density map of the primary shock front in any atmospheric laser desorption experiment. ${ }^{52,54,55}$

Here, we apply time-resolved digital interference microscopy (DIM) to investigate the PIRL ablation of liquid water under DIVE conditions as a function of laser fluence and demonstrate the imaging method's effectiveness for retrieving the refractive index and density reconstructions of laser ablation plumes. The imaging setup was simplified to only include a single long working distance microscope objective while still yielding sufficient spatial resolution. Recording the hologram in the microscope objective's image plane makes it particularly easy to monitor the sample and perform any adjustments to the optics without the need for on-line image processing. Image acquisition and processing were automated to a degree that made the analysis of a large number of snapshots feasible. Reconstruction of the shock front density was achieved by using a modified implementation of the 
well-known Abel-inversion in combination with the Gladstone-Dale law. ${ }^{56}$ As a comparison, the shock front density was also estimated from the plume expansion velocity. To this end, the time evolution of the primary shock front radii was fit using the Taylor-Sedov (TS) point explosion model $^{57-59}$ with a variable exponent, a model which is often applied to the plume expansion in laser ablation when the observed propagation distances are similar to the laser spot diameter. ${ }^{60-62}$ We utilize an order of magnitude shorter pulse duration (10 ps) than previously characterized for IR water desorption and identify discrete fluence dependent ablation regimes. ${ }^{18}$ Quantitative data of the time evolution of the shock-front velocity are presented to aid future modeling approaches.

\section{METHODS}

\section{A. Laser desorption}

Distilled water was laser desorbed under DIVE conditions using 10 ps pulses at a wavelength of $3 \mu \mathrm{m}$ (PIRL-APLQ3000, Attodyne Inc., Canada). The beam was spatially filtered and slightly expanded in a telescope assembly. A $50 \mathrm{~mm}$ focal length $\mathrm{CaF}_{2}$ lens ( $\mathrm{L}_{1}$ in Fig. 1) was used to focus the light onto the sample under normal incidence, yielding a near-Gaussian, slightly elliptical spot with $1 / \mathrm{e}^{2}$ diameters of $(43 \pm 7) \mu \mathrm{m}$ perpendicular and $(38 \pm 5) \mu \mathrm{m}$ parallel to the illumination laser axis as determined by knife edge measurements. The PIRL pulse energy was monitored on an absorptive detector (Thorlabs PDA20H-EC) during ablation and an energy assigned to each image to reduce the influence of pulse energy fluctuations on data analysis, as is described in more detail in the supplementary material. The peak fluence in the focus center was varied between $(0.16 \pm 0.06) \mathrm{J} \mathrm{cm}^{-2}$ and $(4.7 \pm 1.0) \mathrm{J} \mathrm{cm}^{-2}$ (corresponding to pulse energies between $(1.0 \pm 0.3) \mu \mathrm{J}$ and $(30.0 \pm 0.8) \mu \mathrm{J})$ using a variable neutral density filter at the laser output $\left(\mathrm{ND}_{\mathrm{V}}\right)$. The stated standard errors stem from the power calibration procedure and the spot size uncertainty. A stable, curved water surface was maintained on top of a $100 \mu \mathrm{m}$ inner diameter capillary by slowly replenishing the water using a syringe pump (New Era Pump Systems NE-1002X with Hamilton $25 \mu \mathrm{L}$ \#702). The water surface position was held within $30 \mu \mathrm{m}$ using feedback from the camera image to ensure stable focus conditions.

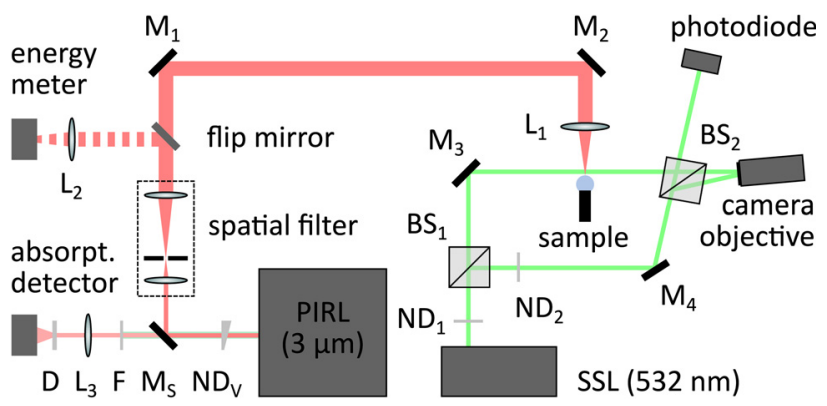

FIG. 1. Beam paths for the picosecond infrared laser (PIRL) used for ablation and the illuminating solid state laser (SSL). $\mathrm{L}_{1-3}$ : calcium fluoride lenses, $\mathrm{M}_{1-4}$ : silver mirrors, $\mathrm{BS}_{1-2}$ : beam splitters, D: diffuser, $\mathrm{F}$ : bandpass filter, $\mathrm{M}_{\mathrm{S}}$ : beam splitting mirror, $\mathrm{ND}_{1-2}$ : neutral density filters, and $\mathrm{ND}_{\mathrm{V}}$ : variable neutral density filter.

\section{B. Digital interference microscopy}

Various configurations and image reconstruction methods are known for DIM. ${ }^{41}$ Here, time resolved digital interference microscopy was implemented in a pump-probe setup schematically depicted in Fig. 1: a diode-pumped passively Q-switched solid state laser (FDSS 532-Q, CryLas GmbH, Berlin, Germany) was used to produce green light pulses with a duration of $1.4 \mathrm{~ns}$, short enough to prevent motion blurring within one image acquisition. The beam was split in an off-axis Mach-Zehnder configuration into a collimated object beam illuminating the sample and a reference beam traveling a similar distance through an open space. Both beams were recombined at an angle (at $\mathrm{BS}_{2}$ in Fig. 1), and a long working distance microscope objective with variable magnification (Optem Fusion, Qioptiq, Waltham, USA) was placed so that a sharp image of the object (the water splash following a laser ablation event) was formed on the monochrome CCD detector (DMK 23U274, The Imaging Source, Bremen, Germany, $1600 \times 1200$ pixels, $4.4 \mu \mathrm{m} \times 4.4 \mu \mathrm{m}$ pixel size). Because both beams traversed the same objective, their wavefronts were intrinsically curvature matched (except for lens-aberrations). Due to the narrow spectrum of the solid state illumination laser (SSL), the coherence length was large enough that no special care had to be taken to ensure proper interference at the detector. Timing synchronization of the SSL pulse and camera shutter relative to the ablating PIRL was implemented with a picosecond-precision delay generator (SRS DG645). To account for any timing jitter between the PIRL and the SSL, stray light from the PIRL's internal pump pulse (at $1 \mu \mathrm{m}$ ) and the part of the SSL reference beam that was transmitted at the second beam splitter $\left(\mathrm{BS}_{2}\right)$ were captured on separate fast photodiodes, the signals recorded on an oscilloscope (Agilent DSO-X 3024A) and saved on a computer using LabVIEW. The exact delay between the two pulses was determined with a precision on the order of the signal rise times $(<2 \mathrm{~ns})$.

The details of the image reconstruction are outlined in the supplementary material. In short, the angular spectrum method $^{41,63}$ is used, whereby the phase shift $\varphi$ is recovered from the recorded interferogram through filtering with a simple rectangular window in the Fourier-domain. Any remaining wavefront curvature not associated with the object is removed from the reconstructed phase by using a double exposure scheme ${ }^{44,64}$ in which a second image of the unperturbed water surface is taken fractions of a second before the actual image of the laser impact, and the background phase is subtracted. Minimizing the delay between both acquisitions is beneficial to suppress the movement of the water surface and changes in the background phase (e.g., by convection). A two-dimensional phase unwrapping algorithm is applied to remove any $2 \pi$ discontinuities from the resulting phase ${ }^{65}$ and the phase is offset so that it is zero in the unperturbed regions in the top edge of the image.

The spatial resolution of the technique differs between the directions parallel and perpendicular to the interference fringes. In the former, the resolution is equal to that of the microscope objective $(2.9 \mu \mathrm{m}$ at maximum magnification setting), whereas in the latter, it is determined by the 
interplay of magnification, pixel size, and recombination angle $\alpha$. Because we chose both the object and reference beam to traverse the same objective lens, the limited acceptance angle $\theta=\arcsin (\mathrm{NA})$ (with NA $=0.12$ ) of the microscope objective limits the maximum recombination angle to $\alpha=2 \theta$. This in turn limits the achievable spatial resolution: band-pass filtering in the Fourier domain removes all spatial frequencies larger than $u_{\max }=u_{0} / 2$, where $u_{0}$ is the spatial frequency of the unperturbed fringe pattern. This reduces the resolution of the resulting phase map to $\delta x=1 / u_{\max }$ $=2 M / k \sin \alpha$, which is the object size covered by two fringe periods $\Delta x=1 / u_{0}$. The largest $\alpha$ was achieved by aligning both beams symmetrically with respect to the objective, resulting in a fringe spacing of about 6 pixels or $\delta x=6 \mu \mathrm{m}$. Usually a slightly larger fringe spacing was chosen for better illumination, and a resolution of about $\delta x \approx 7.5 \mu \mathrm{m}$ at a magnification of $M=8.8$ was verified by imaging a resolution target (Thorlabs 1951 USAF Test Target). The signal quality is also limited by noise, which is in part due to an imperfect separation of the DC and AC spectra and in part caused by changes in the optical path length between the background and the sample image, e.g., by vibrations or convection. As a measure of phase accuracy, the noise level was determined by calculating the standard deviation of an unperturbed area of different background-subtracted phase images ${ }^{45}$ which yielded values of $\sigma<0.018 \approx \pi / 170$.

\section{Abel inversion and density maps}

The phase images $\varphi(x, y)$ obtained using DIM permit the recovery of the refractive index distribution and ultimately the density distribution in the object beam. However, because the images acquired in DIM represent a twodimensional projection of a three-dimensional object, the recovery of the $3 \mathrm{D}$ distribution is non-trivial. One way to approach the loss of depth-information is to assume a cylindrical symmetry of the object. This is well-justified in the case of laser-ablation plumes from a surface with the same symmetry, such as the locally flat droplet surface we are concerned with here. With the $y$-axis being the axis of symmetry, we can thus write

$$
\varphi(x, y)=\frac{4 \pi}{\lambda} \int_{|x|}^{\infty} \frac{r \Delta n(r, y)}{\sqrt{r^{2}-x^{2}}} \mathrm{~d} r .
$$

The inversion of Eq. (1) is a well-known problem referred to as the inverse Abel transformation. From the plethora of possible inversion algorithms, we chose the Gaussian Basis-Set Expansion Method, ${ }^{66}$ which is commonly employed to analyze velocity map imaging data, as it is designed to reproduce the sharpest features, has a large dynamic range, and handles noise well without introducing spurious artifacts. The corresponding section of the pyabel python package ${ }^{67}$ was implemented in Matlab and thoroughly tested against the original algorithm before use.

The Gladstone-Dale relation was used to determine the density in the primary shock front from its refractive index. ${ }^{56}$
The relative density of the compressed ambient air can be calculated as

$$
\frac{\rho(x, y)}{\rho_{0}}=1+\frac{\Delta n(x, y)}{n_{0}-1} .
$$

The ambient refractivity $\left(n_{0}-1\right)=277 \times 10^{-6}$ was determined based on the updated Edlén equation ${ }^{68}$ and the values of air pressure, temperature, humidity, and $\mathrm{CO}_{2}$ content on the day of the experiment.

\section{RESULTS AND DISCUSSION}

\section{A. Phase and amplitude images}

Images of the plume expansion were acquired for laser fluences between 0.16 and $4.7 \mathrm{~J} \mathrm{~cm}^{-2}$ and for time delays between 0 and $1000 \mathrm{~ns}$, which was the time scale on which the plume front would leave the camera's field of view. Coarse time steps were used for longer time delays. FFTfiltering and background-subtraction were performed on each image pair to yield both the reconstructed amplitude and phase. The analysis of the collected phase images revealed three different ablation regimes. At laser fluences below $F=0.5 \mathrm{~J} \mathrm{~cm}^{-2}$ ( $3 \mu \mathrm{J}$ pulse energy), no disturbance of the water surface was observed. At fluences slightly above this ablation threshold, a hemispherical shock wave was observed to emanate from the location of the laser impact at the water surface [Figs. 2(a) and 2(b)]. In accordance with
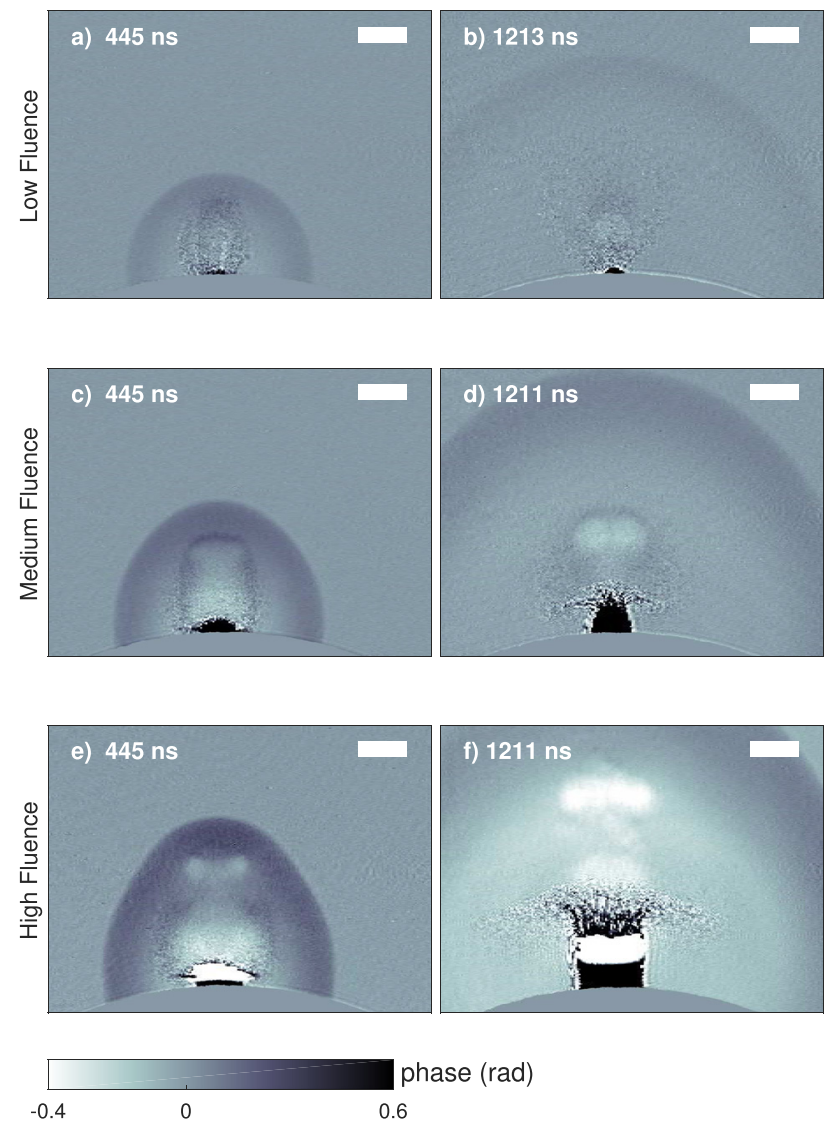

FIG. 2. Reconstructed phase images showing different ablation regimes for low $\left(1 \mathrm{~J} \mathrm{~cm}^{-2}\right)$, intermediate $\left(2 \mathrm{~J} \mathrm{~cm}^{-2}\right)$, and high $\left(>3 \mathrm{~J} \mathrm{~cm}^{-2}\right)$ laser fluences. Time stamps are relative to the time of laser incidence, and the scale bar is $100 \mu \mathrm{m}$. 

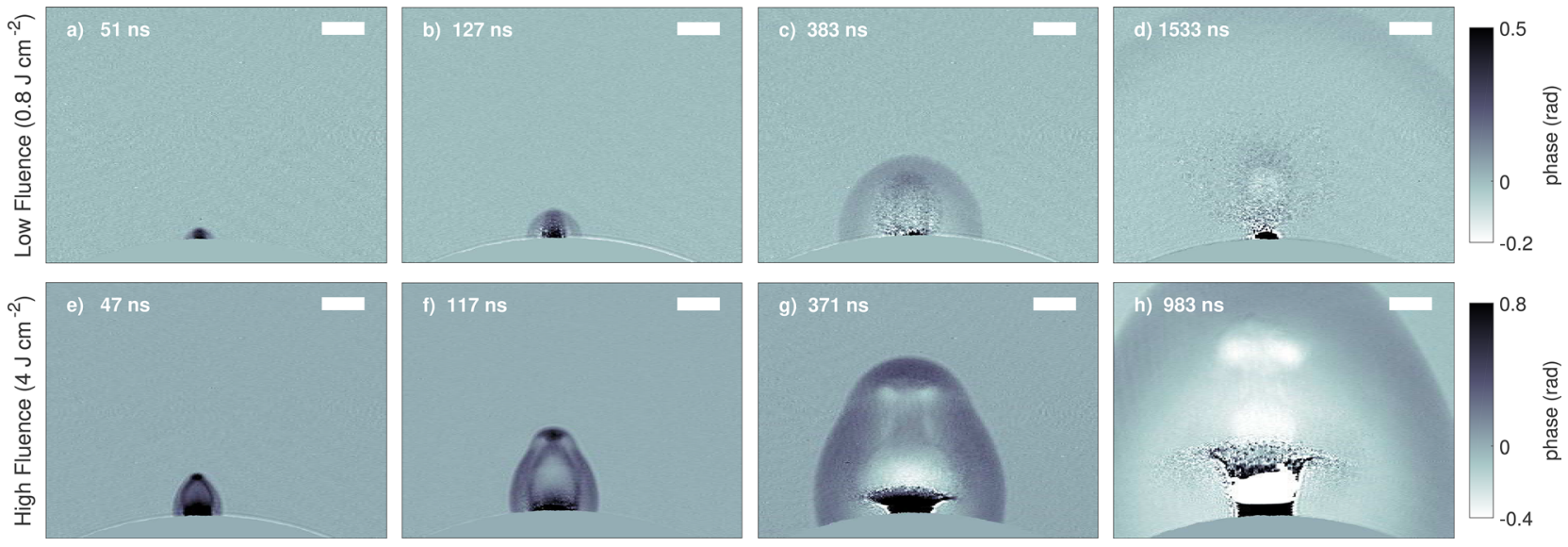

FIG. 3. Reconstructed phase images of the early stage plume expansion for the low [(a)-(d), $\left.0.8 \mathrm{~J} \mathrm{~cm}^{-2} / 5 \mu \mathrm{J}\right]$ and high $\left[(\mathrm{e})-(\mathrm{h}), 3.9 \mathrm{~J} \mathrm{~cm}^{-2} / 25 \mu \mathrm{J}\right]$ fluence regimes. Time stamps are relative to the time of laser incidence, and the scale bar is $100 \mu \mathrm{m}$.

previous studies, ${ }^{62,69}$ this primary shock wave (PSW) is identified as an acoustic wave resulting from a sudden compression of the ambient air as the heated water begins to expand. Further inside the plume, a denser region containing the target material could be distinguished, the border of which was the contact front (CF). For low fluences, the region condensed into a cloud of water vapor and small droplets within the first $100 \mathrm{~ns}$. At intermediate fluences [Figs. 2(c) and 2(d)], the plume consisted of fewer droplets and instead showed a stronger contact front, indicating that a larger fraction of the ejected material reached the evaporation enthalpy. The plume expansion at the highest employed fluences [Figs. 2(e) and 2(f)] was characterized by a turbulent propagation of the central part of the contact front. The higher the laser fluence, the larger the ratio of initial velocities in normal and lateral directions, which did not only lead to a forward peaked propagation of the CF but also for high fluences produced a PSW shape which was elongated along the propagation direction. For all fluences, a recoil-induced displacement of liquid water slowly expanded outwards directly above the water surface. Note that the phase jumps in the regions of liquid water in Figs. 2(e) and 2(f) are due to insufficient light transmission and/or a phase gradient that exceeds the system's resolution and are thus an artifact of the imaging method. At later times [ $>1 \mu \mathrm{s}$, see Figs. 2(b), 2(d), and 2(f)], a low-density region could be observed inside the vapor/droplet cloud, constituting a rarefaction wave following the explosive ablation. Figures 3 and 4 show the time evolution of the expansion for high and low fluences by means of the reconstructed phase and amplitude data, respectively. While for low fluences a high (optical) density region at the top of the plume, i.e., between PSW and CF, dissipates quickly, it is well visible and not separating from the PSW front for the first few hundred ns for intermediate fluences. For the highest fluences, it protrudes outwards, distorting the PSW's shape and making it more elliptical in nature. We observe the threshold for the formation of this plume shape to fluctuate around $2.5 \mathrm{~J} \mathrm{~cm}^{-2}$ to $3.6 \mathrm{~J} \mathrm{~cm}^{-2}$. In the reconstructed amplitude images, the contrast of the gas-phase portions of the plume is lower than for the phase images. However, they serve well to identify the liquid-phase portions of the plume. Additionally, a region of faint positive amplitude change is visible behind each shock front, indicating high temperatures of the shocked gas. The luminescence is especially pronounced for the high density region at the top of the plume. As shown in Figs. 4(a) and 4(e), the
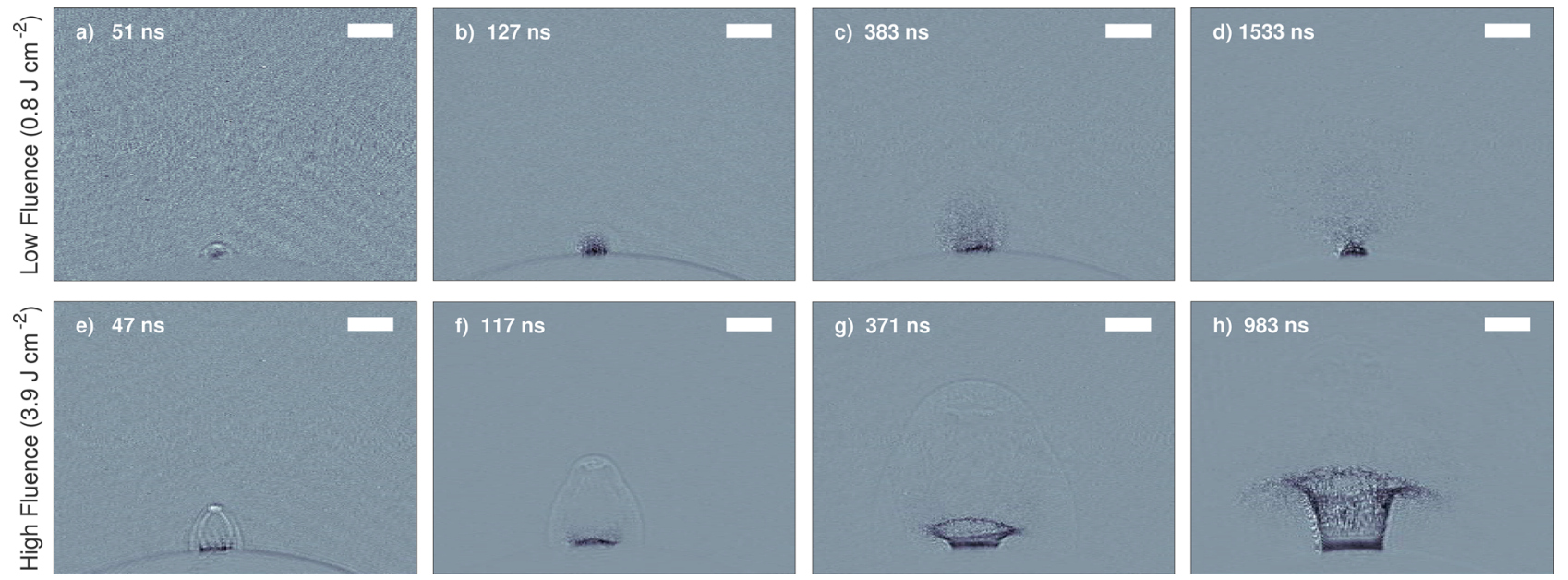

FIG. 4. Reconstructed amplitude images of the early stage plume expansion for the low [(a)-(d), $\left.0.8 \mathrm{~J} \mathrm{~cm}^{-2} / 5 \mu \mathrm{J}\right]$ and high $\left[(\mathrm{e})-(\mathrm{h}), 3.9 \mathrm{~J} \mathrm{~cm}^{-2} / 25 \mu \mathrm{J}\right]$ fluence regimes. Time stamps are relative to the time of laser incidence, and the scale bar is $100 \mu \mathrm{m}$. 
luminescence is visible at short time delays for all fluences (except possibly right at the ablation threshold where it was too faint to observe). While it dissipates quickly for low fluences, for high fluences, the high-density region moves rapidly, creating two ring-vortices and a turbulent updraft of water vapor in its wake.

Figure 5 shows the ablation dynamics on a longer, microsecond timescale for the high $\left(\approx 3.90 \mathrm{~J} \mathrm{~cm}^{-2} / 25 \mu \mathrm{J}\right)$ and low $\left(\approx 0.78 \mathrm{~J} \mathrm{~cm}^{-2} / 5 \mu \mathrm{J}\right)$ laser fluence regimes. At these delays, the primary shock front was no longer in the field of view, and the dynamics were fully composed of droplets and water surface movement. An accurate phase determination is difficult for liquid materials, as is evidenced by the aforementioned artifacts in Figs. 2(e) and 2(f) and also Fig. 3(h). Therefore, we only show the reconstructed amplitudes (i.e., the background-subtracted absolute value of the FFT filtered interference pattern). For high fluence, the initial water column that is visible at $1 \mu \mathrm{s}$ in Fig. 3(h) contracts due to underpressure and surface tension as it propagates away from the water surface, and it moves slower than the surrounding cloud of droplets. A second cavitation bubble forms at its base, as shown in Fig. 5(e), similarly contracting to form another liquid water column. At around $300 \mu \mathrm{s}$, no further expulsion of the material was observed, although the water surface still showed slight oscillations. For low fluence, the amount of the ejected material was much smaller, and thus, less recoil occurred. The water column was less pronounced or even absent, and the desorption event subsided much faster $(50 \mu$ s at $5 \mu \mathrm{J})$.

Some amount of blurring due to out-of-focus portions of the plume is expected given that the microscope objective's depth of field was only around $40 \mu \mathrm{m}$. This is especially noticeable for ejected droplets visible at later time delays, which show distinct diffraction rings. The spatial resolution for the outermost part of the plume, however, is not significantly altered because, due to its spherical shape, the plume front does not extend far beyond the focal plane in these regions. Spatial resolution is thus mainly affected in the center of the ablation plumes where the extension perpendicular to the focal plane is large.
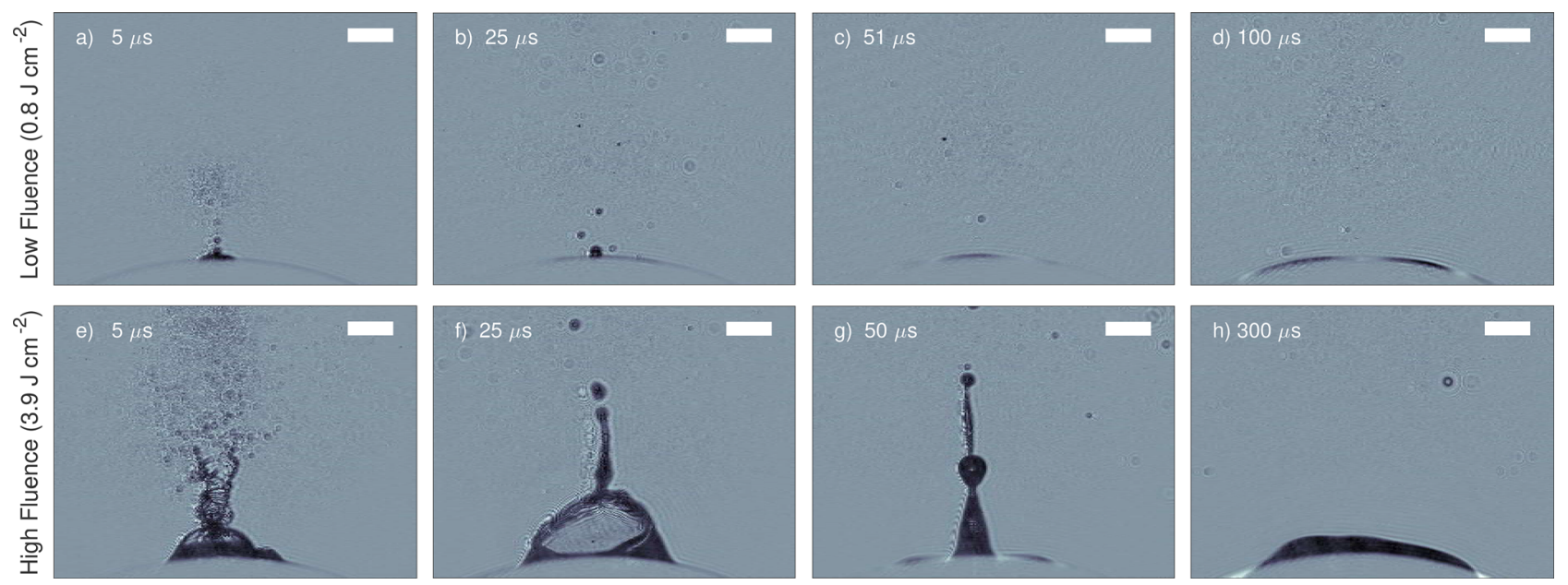

FIG. 5. Reconstructed amplitude images of the plume expansion at later times for the low [(a)-(d), $\left.0.8 \mathrm{~J} \mathrm{~cm}^{-2} / 5 \mu \mathrm{J}\right]$ and high $\left[(\mathrm{e})-(\mathrm{h}), 3.9 \mathrm{~J} \mathrm{~cm}^{-2} / 25 \mu \mathrm{J}\right]$ fluence regimes. Time stamps are relative to the time of laser incidence, and the scale bar is $100 \mu \mathrm{m}$. 
The cause for the observed differences compared to nanosecond ablation is not necessarily clear. A similar but more pronounced fast expansion of the central plume, which tends to form a triangular primary shock front, is seen for femtosecond pulses in the laser ablation of metals because of the optical breakdown of the ambient air in the focal region and the formation of a plasma filament. ${ }^{72}$ For longer pulses, plasma formation due to multiphoton absorption becomes less significant, but a plasma can still form due to cascading ionization if sufficient seed charges are created. We calculate a maximum peak irradiance of $5 \times 10^{11} \mathrm{~W} \mathrm{~cm}^{-2}$ at the center of the laser focus, which at visible and near-infrared wavelengths is sufficient to initiate cascading ionization and to ultimately lead to plasma formation, ${ }^{73}$ but not necessarily at mid-IR wavelengths. The conditions in the present study can be shown to be slightly below the threshold for the breakdown of water by solving the commonly employed rate equation for the free electron density ${ }^{74}$ with parameters adjusted to DIVE ablation, and an optical breakdown seems unlikely to be responsible for the observed plume shape considering that the same shape was observed by Franjic et al. for much lower fluences and for a pulse duration a factor of 10 larger, yielding irradiances far below the theoretical onset of cascading ionization.

Laser breakdown under ambient conditions for long pulse durations can also be discussed in the framework of laser absorption waves. ${ }^{75}$ Similar plume shapes have been observed for the near-IR ablation of metals and polymers with nanosecond lasers and attributed to a laser-supported detonation or laser-supported combustion waves, in which the initial part of the laser pulse creates free charges, for example, by thermal ionization behind the emerging supersonic shock wave, which then absorb the later parts of the pulse. ${ }^{76,77}$ However, these models predict the generation of a hot plasma, and no plasma spark was observed by eye even for kilohertz operation of the laser system, just as no plasma spark was observed in a previous study ${ }^{78}$ with similar ablation conditions $(2.92 \mu \mathrm{m}$ wavelength, 19 ps pulse duration, and $30 \mu \mathrm{m}$ spot diameter) even at a higher peak irradiance of $3.4 \times 10^{12} \mathrm{~W} \mathrm{~cm}^{-2}$ (peak fluence of $65 \mathrm{~J} \mathrm{~cm}^{-2}$ ). Even though thermal ionization of the leading portion of the plume is possible in the present study, we do not observe a significant contribution of the free charge density to the shock front's optical density. If present, free electrons give a negative contribution to the refractive index $n=\sqrt{1-\frac{N_{e}}{N_{c}}}$, where $N_{e}$ is the electron density and $N_{c}$ is the critical density corresponding to a plasma frequency equal to the illumination laser frequency, and would thus reduce the phase shift observed in the experiment. ${ }^{53}$ The protrusion of the PSW is seen as an optically dense region, indicating that the density of free electrons is far below the critical value for plasma generation of $N_{e} \approx 10^{18}-10^{21} \mathrm{~cm}^{-3}$ for all recorded images. ${ }^{74}$

We conclude that the high density region at the top of the plume constitutes a dense cloud of hot water vapor which is ejected with high velocity and almost no time lag with respect to the PSW. Additional momentum is possibly gained by thermal expansion, leading to a distortion of the PSW for high fluences, whereas the material cloud dissipates more quickly for low fluences so that no distortion can be observed. The protrusion might not have been observed for the nanosecond ablation performed by Apitz et al. even though a phase explosion with complete vaporization of the surface layer was achieved because for pulse durations above the stress confinement regime, the primary shock front and the contact front separate before the phase explosion is complete. The PSW was thus unaffected by the CF's protrusion and kept its hemispherical shape. The fluence threshold for which a distortion could be observed in the present study is high compared to the results by Franjic et al., most likely because dissipation is more efficient for the smaller laser spot diameter employed here. Additionally, a slight increase in the optical penetration depth into the water surface could potentially be caused by photo-bleaching of the water absorption band as the energy was deposited on the timescale of vibrational relaxation, ${ }^{79}$ which would result in a lower energy density of the surface layer.

\section{B. Blast-wave theory and plume velocity}

Digital interference microscopy allows for the extraction of additional quantitative physical information from the recorded images. Figure 6(a) shows a density map based on the data shown in Fig. 3(f). In order to reduce noise, the left and right sides of each phase image were averaged and slightly smoothed using a $5 \mu \mathrm{m} \times 18 \mu \mathrm{m}$ Wiener filter before the Abel inversion was performed. The density map is thus symmetric. The contrast between high- and low-density regions inside the plume is considerably better than in the projected phase image, which simplifies subsequent image processing. Three distinct regions of the plume were identified for each recorded image using a custom Matlab image processing script. The regions are marked in the right half of Fig. 6(a). The rising front of the PSW was found by simple thresholding along radial paths pointing towards the point of laser incidence (blue). Based on this position, the peak of the PSW could be located (orange). Identifying the CF by thresholding was difficult due to its complex shape, which was often granular and ill-defined. It was thus only performed in the near-normal direction and for a limited time range. The density along the PSW front (orange curve) is shown in Fig. 6(b) as a function of the angle from the surface
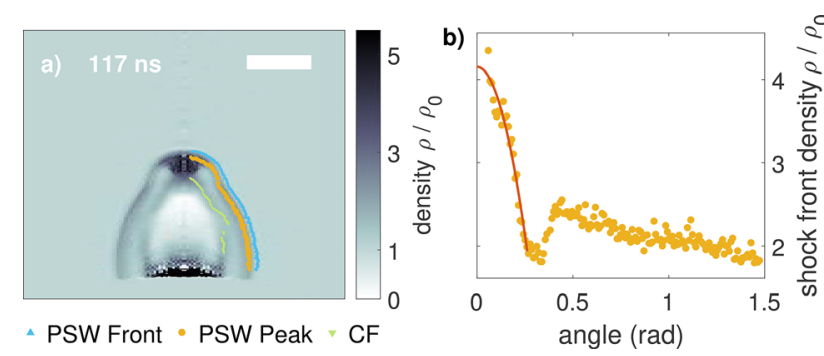

FIG. 6. Density reconstruction of the PSW in units of the ambient air density $\rho_{0}$. (a) Density reconstruction of data shown in Fig. 3(f). The image is symmetric. The right side shows the positions of the start of the PSW (blue), the peak of the PSW (orange), and the start of the CF (green) as identified by a Matlab script. (b) Density at the peak of the PSW as a function of the angle from the surface normal. A quadratic, symmetric fit is applied to extrapolate the density at zero angle (orange line). 
normal. The pressure in the normal direction was found to be very high, which we attribute to the overlap between the PSW front with the high density region separating from the contact front. To the side of this region (angle $\approx 0.4 \mathrm{rad}$ ), the pressure dropped by a factor of two as the lateral velocity of the bulge was much lower, leading to a much fainter pileup of ambient air. The pressure rise for larger angles resembles the PSW front of the hemispherical shock, which again expanded with high velocity in the normal direction. The overlaid curve in Fig. 6(b) is a quadratic fit to extrapolate the density in the normal direction, which could not be directly obtained because of the center-line noise resulting from the Abel-inversion. A quadratic fit was chosen as an approximation of the real angular density profile, which reflects the forward peaked elliptic expansion velocity profile.

Additional quantitative information was obtained from the shape and velocity of the primary shock front. The TaylorSedov (TS) blast wave theory ${ }^{57}$ is commonly employed to model the plume expansion and extract a conversion efficiency from laser pulse energy to shock-wave-energy. ${ }^{34,69,80}$ It models the radius of a (hemi-)spherical shock wave as a function of time under the assumption that the blast energy is deposited instantaneously and in a point source, that the mass of the swept-up air is much greater than that of the ablated material, and that the shock front pressure $p_{\mathrm{s}}$ is much higher than the ambient pressure $\left(p_{\mathrm{s}} / p_{0}>10\right)$. In an extension of the model to arbitrary dimensionalities, namely, planar, cylindrical, and spherical shock waves $\left(\nu=1,2,3\right.$ respectively), ${ }^{59}$ the shock front radius $R$ may be written as

$$
R(t)=\xi_{\nu}\left(\frac{E_{0}}{\rho_{0}}\right)^{\frac{1}{\nu+2}} \frac{2}{t^{\nu+2}}
$$

where $\xi_{\nu}$ is a function of the specific heat ratio $\gamma$ and the dimensionality $\nu, E_{0}$ is the released energy, $\rho_{0}$ is the ambient density, and $t$ is the time. The PSW front radii were extracted from the density maps using the Matlab image processing script mentioned previously. The rising front [blue curve in Fig. 6(a)] was used as a position marker as it was identified most reliably and showed no significant difference to the peak position. The shock front positions in normal and lateral directions with respect to the center point on the water surface were fitted with the TS model in the form $R(t)=A\left(t-t_{0}\right)^{q}$, with two fit parameters $A$ and $q$ and a global offset in delay time determined by a three-parameter fit of the PSW positions in the normal direction. Figure 7(a) shows an exemplary fit for a pulse energy of $22 \mu \mathrm{J}$. High pressure gradients across the water surface cause a fast initial expansion in the normal direction, while at later times, the expansion is almost spherical. The results of the TS model for different fluences for the expansion in the normal direction are shown in Fig. 7(b). The energy coefficient $A$ shows a linear increase with fluence and is thus, in conflict with the earlier observations, not an indication of a transition in the ablation mechanism. The fitted exponent $q$ decreases from an almost linear plume expansion $R \propto t$ at low fluences to an expansion with cylindrical shock-wave-character $(\nu=0.5)$ at high fluences. Although the coefficient $A$ does not show any transition, the data for $q$ indicate two regimes. For low
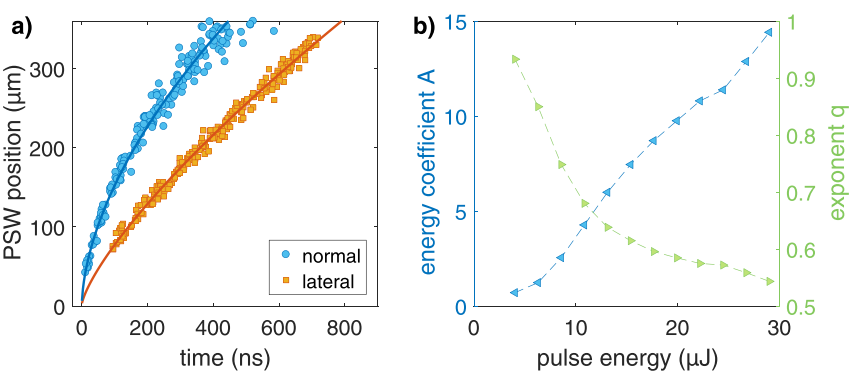

FIG. 7. Fitting the PSW expansion with the Taylor-Sedov model. (a) Fit for both normal and lateral expansion directions at a pulse energy of $22 \mu \mathrm{J}$. (b) Fit coefficients $A$ (triangles pointing left) and $q$ (triangles pointing right) as a function of pulse energy.

pulse energies, the exponent decreases strongly with rising pulse energy, while the slope is much less for energies above $\approx 15 \mu \mathrm{J}$, which is close to the energy at which a significant PSW protrusion becomes visible. The results for the lateral expansion (not shown) convey similar trends for $A$ and $q$ but are more noisy and less distinctive. Because the exponent $q$ was used as a fit parameter, the coefficient $A$ holds no quantitative information and the mechanical energy $E_{0}$ released in the shock cannot be determined. ${ }^{62}$ The adaption of the exponent was necessary, however, because the laser ablation plume changes its shape from planar to elongated along the surface normal and only becomes spherical at later times, which does not fulfill the requirements of the TS model. Nonetheless, the adapted model fits the shock front positions very well and thus serves to extract the instantaneous shock front velocity. These are shown in Fig. 8(a) for different pulse energies and compared to the speed of sound marked by a red line. The plume front strongly decelerates in the first few hundred ns and reaches Mach 1 at around $1 \mu$ s for all fluences shown. Because of the strong deceleration, it is difficult to assign an initial velocity, which will critically depend on the measurement's time resolution. We approximate the temperature $T_{s}$ of the compressed background gas at a time delay of $100 \mathrm{~ns}$ and for the high and low fluence regimes $\left(0.8 \mathrm{~J} \mathrm{~cm}^{-2} / 3.9 \mathrm{~J} \mathrm{~cm}^{-2}\right)$, similar to the images shown in Figs. 3 (b) and 3(f), by using the Rankine-Hugoniot jump conditions of the shock wave expansion as ${ }^{81}$

$$
\frac{T_{s}}{T_{0}}=\frac{\left(M_{s}^{2}+5\right)\left(7 M_{s}^{2}-1\right)}{36 M_{s}^{2}},
$$

where $T_{0}$ is the ambient temperature, $M_{s}=\left(u_{s} / c_{0}\right)$ is the shock wave's Mach number, $c_{0}$ is the speed of sound in the ambient air, and $\gamma=1.4$ has been assumed to model air as an ideal gas. The estimated shock front temperatures after $100 \mathrm{~ns}$ are $680 \mathrm{~K}$ and $380 \mathrm{~K}$ for high and low fluences, respectively.

Knowledge of the shock front velocities $u_{s}$ also facilitates the calculation of the shock front density $\rho_{s},{ }^{81}$ making it possible to compare the values obtained through Abel inversion of the phase information. Again assuming an ideal and calorically perfect gas, the density reads

$$
\frac{\rho_{s}}{\rho_{0}}=\frac{6 M_{s}^{2}}{5+M_{s}^{2}},
$$

where $c_{0}$ is the speed of sound in the ambient air. The resulting evolution of the PSW front density is shown in Fig. 8(b) 

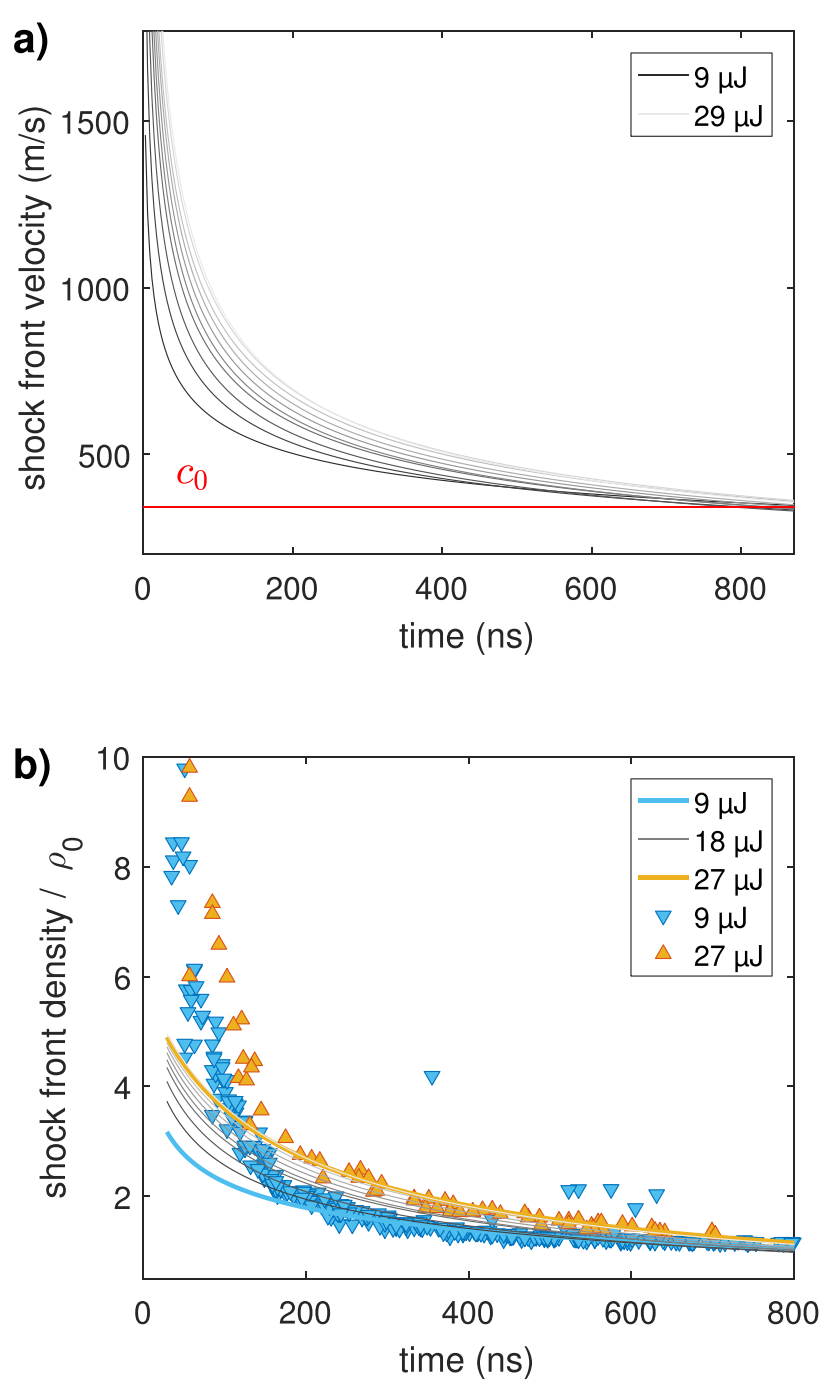

FIG. 8. (a) Shock front velocities in the normal direction resulting from the Taylor-Sedov fits for different pulse energies. Lighter colors mean higher pulse energies. The red line indicates the speed of sound in ambient air $c_{0}$. (b) To compare the two calculations, lines show the PSW density evolution computed from the shock front velocities, while markers show the centerline density extrapolated from the density reconstructions as shown in Fig. 6(b).

as a series of line plots for different pulse energies. They overlap very well with the results from the density maps, plotted as scatter plots for a high and a low pulse energy, for delay times $>200 \mathrm{~ns}$. For shorter time delays, the density maps show very high values up to 10 times the ambient density, violating the prediction of the shock-wave-model that in the high pressure limit, the maximum shock front density is $(\gamma+1) /(\gamma-1)=6$ times the ambient density for $\gamma=1.4$ (ideal gas). This indicates that either the specific heat ratio $\gamma$ deviates significantly from its ideal value for the pressures found at the shock front or that there is an admixture of ablated water vapor at high density. The latter is very likely the case given that the PSW front and CF have not yet separated for early times.

\section{CONCLUSION}

We demonstrated the application of time resolved offaxis digital interference microscopy to the plume expansion of picosecond infrared laser desorption (PIRL) of water under stress confinement conditions (DIVE). The imaging setup uses only one microscope objective and is simple to align and is yet capable of producing high-contrast images for the micrometer-sized and highly transparent ablation plumes, thus being well suited to characterize the shock front evolution with good spatial and temporal resolution. After proper background subtraction, the method shows very little interference noise, facilitating short-pulsed laser sources for illumination, which introduce interference artifacts based on temporal coherence in other flash-photography approaches. The obtained contrast is proportional to the optical path length difference between the object and the ambient air and can thus yield additional information to that obtained by techniques based on refraction. The retrieved phase maps have been used to estimate the shock front density and could alternatively yield the shock front velocity with a single image acquisition [rearranging Eq. (5) and using $\rho_{s}$ from the Abel inversion] as compared to the usually performed fit of the velocity extracted from several consecutive snapshots [Fig. 8(a)].

We presented a systematic study of the temporal plume evolution with laser fluence and identified different ablation regimes. The material emission was strongly peaked in the forward direction as observed in previous studies and under DIVE conditions is led by a hot and expanding vapor cloud. For large fluences, this vapor cloud took several hundred ns to cool down, and it moved fast enough to cause a significant distortion of the primary shock front. The reconstructed refractive index field confirms that no significant density of free charges was present after several tens of nanoseconds after laser incidence, i.e., the ablation was not plasma mediated. For atmospherically coupled mass spectrometry, the fluence regime close to the ablation threshold can be considered to be most suitable because it results in a fine mist of droplets with low internal temperature. The hot vapor cloud, however, could be of special interest to spectroscopy or in-vacuo mass spectrometry.

\section{SUPPLEMENTARY MATERIAL}

See supplementary material for a more detailed description of the phase reconstruction method and the pulse energy calibration procedure.

\section{ACKNOWLEDGMENTS}

The authors thank F. Tellkamp and M. Kollewe for technical support, K. Strahlendorff for her aid in setting up the first version of the experiment, and B. Reber for implementing the Abel Inversion algorithm.

R. J. D. Miller is the author of Patent U.S. 2010/ 0213367 A1 describing the DIVE process and the cofounder of Attodyne Inc. The other authors declare no competing financial interests.

This work was supported by the Max-Planck Society and is part of Project SUREPIRL funded by the European Research Council (ERC-AdG-2011-291630).

${ }^{1}$ J. J. Corr, P. Kovarik, B. B. Schneider, J. Hendrikse, A. Loboda, and T. R. Covey, J. Am. Soc. Mass Spectrom. 17, 1129 (2006). 
${ }^{2}$ M. Ith, H. Pratisto, H. J. Altermatt, M. Frenz, and H. P. Weber, Appl. Phys. B 59, 621 (1994).

${ }^{3}$ D. Palanker, A. Vankov, J. Miller, M. Friedman, and M. Strauss, J. Appl. Phys. 94, 2654 (2003).

${ }^{4}$ I. Apitz and A. Vogel, Appl. Phys. A 81, 329 (2005).

${ }^{5}$ D. B. Geohegan, Thin Solid Films 220, 138 (1992).

${ }^{6}$ A. Sambri, S. Amoruso, X. Wang, M. Radovic, F. Miletto Granozio, and R. Bruzzese, Appl. Phys. Lett. 91, 151501 (2007).

${ }^{7}$ S. I. Anisimov and B. S. Luk'yanchuk, Phys. Usp. 45, 293 (2002).

${ }^{8}$ Z. Chen, A. Bogaerts, and A. Vertes, Appl. Phys. Lett. 89, 041503 (2006).

${ }^{9}$ Z. Chen and A. Vertes, Phys. Rev. E 77, 036316 (2008).

${ }^{10}$ E. Nordhoff, A. Ingendoh, R. Cramer, A. Overberg, B. Stahl, M. Karas, F. Hillenkamp, P. F. Crain, and B. Chait, Rapid Commun. Mass Spectrom. 6, 771 (1992)

${ }^{11}$ S. Berkenkamp, C. Menzel, M. Karas, and F. Hillenkamp, Rapid Commun. Mass Spectrom. 11, 1399 (1997).

${ }^{12}$ S. Berkenkamp, Science 281, 260 (1998).

${ }^{13}$ S. Niu, W. Zhang, and B. T. Chait, J. Am. Soc. Mass Spectrom. 9, 1 (1998).

${ }^{14}$ S. J. Lawson and K. K. Murray, Rapid Commun. Mass Spectrom. 14, 129 (2000).

${ }^{15}$ V. V. Laiko, N. I. Taranenko, V. D. Berkout, M. A. Yakshin, C. R. Prasad, H. S. Lee, and V. M. Doroshenko, J. Am. Soc. Mass Spectrom. 13, 354 (2002).

${ }^{16}$ S. Berkenkamp, M. Karas, and F. Hillenkamp, Proc. Natl. Acad. Sci. U. S. A. 93, 7003 (1996)

${ }^{17}$ C. E. Von Seggern, S. C. Moyer, and R. J. Cotter, Anal. Chem. 75, 3212 (2003).

${ }^{18}$ K. Franjic and R. J. D. Miller, Phys. Chem. Chem. Phys. 12, 5225 (2010).

${ }^{19}$ J. Zou, C. Wu, W. D. Robertson, L. V. Zhigilei, and R. J. D. Miller, J. Chem. Phys. 145, 204202 (2016)

${ }^{20}$ L. Ren, W. D. Robertson, R. Reimer, C. Heinze, C. Schneider, D. Eggert, P. Truschow, N.-O. Hansen, P. Kroetz, J. Zou, and R. J. D. Miller, Nanotechnology 26, 284001 (2015).

${ }^{21}$ M. Kwiatkowski, M. Wurlitzer, A. Krutilin, P. Kiani, R. Nimer, M. Omidi, A. Mannaa, T. Bussmann, K. Bartkowiak, S. Kruber, S. Uschold, P. Steffen, J. Lbberstedt, N. Kpker, H. Petersen, R. Knecht, N. O. Hansen, A. Zarrine-Afsar, W. D. Robertson, R. J. D. Miller, and H. Schlter, J. Proteomics 134, 193 (2016).

${ }^{22}$ M. Kwiatkowski, M. Wurlitzer, M. Omidi, L. Ren, S. Kruber, R. Nimer, W. D. Robertson, A. Horst, R. J. D. Miller, and H. Schlter, Angew. Chem. Int. Ed. 54, 285 (2015).

${ }^{23}$ J. Zou, F. Talbot, A. Tata, L. Ermini, K. Franjic, M. Ventura, J. Zheng, H. Ginsberg, M. Post, D. R. Ifa, D. Jaffray, R. J. D. Miller, and A. ZarrineAfsar, Anal. Chem. 87, 12071 (2015).

${ }^{24}$ E. C. Schulz, J. Kaub, F. Busse, P. Mehrabi, H. M. Mller-Werkmeister, E. F. Pai, W. D. Robertson, and R. J. D. Miller, J. Appl. Crystallogr. 50, 1773 (2017).

${ }^{25}$ K. Franjic, M. L. Cowan, D. Kraemer, and R. J. D. Miller, Opt. Express 17, 22937 (2009).

${ }^{26}$ S. Amini-Nik, D. Kraemer, M. L. Cowan, K. Gunaratne, P. Nadesan, B. A. Alman, and R. J. D. Miller, PLoS One 5, e13053 (2010).

${ }^{27}$ H. Petersen, F. Tavakoli, S. Kruber, A. Mnscher, A. Gliese, N.-O. Hansen, S. Uschold, D. Eggert, W. D. Robertson, T. Gosau, S. Sehner, M. Kwiatkowski, H. Schlter, U. Schumacher, R. Knecht, and R. D. Miller, Lasers Surg. Med. 48, 385 (2016).

${ }^{28}$ A. Vogel, J. Noack, G. Httman, and G. Paltauf, Appl. Phys. B 81, 1015 (2005).

${ }^{29}$ L. V. Zhigilei and B. J. Garrison, J. Appl. Phys. 88, 1281 (2000).

${ }^{30}$ L. V. Zhigilei, E. Leveugle, B. J. Garrison, Y. G. Yingling, and M. I. Zeifman, Chem. Rev. 103, 321 (2003).

${ }^{31}$ C. Menzel, K. Dreisewerd, S. Berkenkamp, and F. Hillenkamp, J. Am. Soc. Mass Spectrom. 13, 975 (2002).

${ }^{32}$ A. Rohlfing, C. Menzel, L. M. Kukreja, F. Hillenkamp, and K. Dreisewerd, J. Phys. Chem. B 107, 12275 (2003).

${ }^{33}$ A. Leisner, A. Rohlfing, U. Rhling, K. Dreisewerd, and F. Hillenkamp, J. Phys. Chem. B 109, 11661 (2005).

${ }^{34}$ X. Fan and K. K. Murray, J. Phys. Chem. A 114, 1492 (2010).

${ }^{35}$ S. Amoruso, G. Ausanio, R. Bruzzese, L. Gragnaniello, L. Lanotte, M. Vitiello, and X. Wang, Appl. Surf. Sci. 252, 4863 (2006).

${ }^{36}$ N. Farid, S. S. Harilal, H. Ding, and A. Hassanein, J. Appl. Phys. 115, 033107 (2014).

${ }^{37}$ S. S. Harilal, P. K. Diwakar, M. P. Polek, and M. C. Phillips, Opt. Express 23, 15608 (2015)
${ }^{38}$ A. A. Puretzky, D. B. Geohegan, G. B. Hurst, M. V. Buchanan, and B. S. Luk'yanchuk, Phys. Rev. Lett. 83, 444 (1999).

${ }^{39}$ T. L. Merrigan, D. J. Timson, C. A. Hunniford, M. Catney, and R. W. McCullough, Int. J. Biol. Macromol. 50, 1081 (2012).

${ }^{40}$ A. Vogel, I. Apitz, S. Freidank, and R. Dijkink, Opt. Lett. 31, 1812 (2006).

${ }^{41}$ M. K. Kim, Digital Holographic Microscopy: Principles, Techniques, and Applications, Springer Series in Optical Sciences (Springer-Verlag, New York, 2011).

${ }^{42}$ B. Kemper, D. D. Carl, J. Schnekenburger, I. Bredebusch, M. Schäfer, W. Domschke, and G. von Bally, J. Biomed. Opt. 11(3), 034005 (2006).

${ }^{43}$ C. Canalias, V. Pasiskevicius, F. Laurell, S. Grilli, P. Ferraro, and P. De Natale, J. Appl. Phys. 102, 064105 (2007).

${ }^{44}$ P. Ferraro, S. D. Nicola, A. Finizio, G. Coppola, S. Grilli, C. Magro, and G. Pierattini, Appl. Opt. 42, 1938 (2003).

${ }^{45}$ V. V. Temnov, K. Sokolowski-Tinten, P. Zhou, and D. von der Linde, J. Opt. Soc. Am. B 23, 1954 (2006).

${ }^{46}$ P. Ferraro, S. Grilli, D. Alfieri, S. D. Nicola, A. Finizio, G. Pierattini, B. Javidi, G. Coppola, and V. Striano, Opt. Express 13, 6738 (2005).

${ }^{47}$ C. P. McElhinney, B. M. Hennelly, and T. J. Naughton, Appl. Opt. 47, D71 (2008).

${ }^{48}$ T. Colomb, N. Pavillon, J. Khn, E. Cuche, C. Depeursinge, and Y. Emery, Opt. Lett. 35, 1840 (2010).

${ }^{49}$ F. Merola, P. Memmolo, L. Miccio, R. Savoia, M. Mugnano, A. Fontana, G. D'Ippolito, A. Sardo, A. Iolascon, A. Gambale, and P. Ferraro, Light Sci. Appl. 6, e16241 (2017).

${ }^{50}$ A. Urnieius, N. iaulys, V. Kudriaov, V. Sirutkaitis, and A. Melninkaitis, Appl. Phys. A 108, 343 (2012).

${ }^{51}$ N. Šiaulys, L. Gallais, and A. Melninkaitis, Opt. Lett. 39, 2164 (2014).

${ }^{52}$ K. Choudhury, R. K. Singh, S. Narayan, A. Srivastava, and A. Kumar, Phys. Plasmas 23, 042108 (2016).

${ }^{53}$ D. Breitling, H. Schittenhelm, P. Berger, F. Dausinger, and H. Hgel, Appl. Phys. A 69, S505 (1999).

${ }^{54}$ E. Amer, P. Gren, and M. Sjdahl, J. Phys. D 41, 215502 (2008).

${ }^{55}$ E. Amer, P. Gren, and M. Sjdahl, Opt. Lasers Eng. 47, 793 (2009).

${ }^{56}$ J. H. Gladstone and T. P. Dale, Philos. Trans. R. Soc. London 153, 317 (1863).

${ }^{57}$ G. Taylor, Proc. R. Soc. A 201, 159 (1950).

${ }^{58}$ D. A. Freiwald and R. A. Axford, J. Appl. Phys. 46, 1171 (1975).

${ }^{59}$ G. J. Hutchens, J. Appl. Phys. 88, 3654 (2000).

${ }^{60}$ S. H. Jeong, R. Greif, and R. E. Russo, Appl. Surf. Sci. 127-129, 1029 (1998).

${ }^{61}$ E. de Posada, M. A. Arronte, L. Ponce, E. Rodrguez, T. Flores, and J. G. Lunney, J. Phys.: Conf. Ser. 274, 012078 (2011).

${ }^{62}$ T. A. Schmitz, J. Koch, D. Gnther, and R. Zenobi, J. Appl. Phys. 109, 123106 (2011).

${ }^{63}$ M. Takeda, H. Ina, and S. Kobayashi, J. Opt. Soc. Am. 72, 156 (1982).

${ }^{64}$ V. V. Temnov, K. Sokolowski-Tinten, P. Zhou, and D. von der Linde, Appl. Phys. A 78, 483 (2004).

${ }^{65}$ M. A. Herrez, D. R. Burton, M. J. Lalor, and M. A. Gdeisat, Appl. Opt. 41, 7437 (2002)

${ }^{66}$ V. Dribinski, A. Ossadtchi, V. A. Mandelshtam, and H. Reisler, Rev. Sci. Instrum. 73, 2634 (2002).

${ }^{67}$ PyAbel, Documentation v. 0.7.

${ }^{68}$ K. P. Birch and M. J. Downs, Metrologia 30, 155 (1993).

${ }^{69}$ G. Callies, P. Berger, and H. Hugel, J. Phys. D 28, 794 (1995).

${ }^{70}$ M. A. Mackanos, J. A. Kozub, D. L. Hachey, K. M. Joos, D. L. Ellis, and E. D. Jansen, Phys. Med. Biol. 50, 1885 (2005).

${ }^{71}$ A. Rohlfing, A. Leisner, F. Hillenkamp, and K. Dreisewerd, J. Phys. Chem. C 114, 5367 (2010).

${ }^{72}$ D. Breitling, A. Ruf, and F. Dausinger, Proc. SPIE 5339, 49 (2004).

${ }^{73}$ A. Vogel and V. Venugopalan, Chem. Rev. 103, 577 (2003).

${ }^{74}$ J. Noack and A. Vogel, IEEE J. Quantum Electron. 35, 1156 (1999).

${ }^{75}$ P. E. Nielsen and G. H. Canavan, in Laser Interaction and Related Plasma Phenomena (Springer, Boston, MA, 1974), pp. 177-189.

${ }^{76}$ S.-B. Wen, X. Mao, R. Greif, and R. E. Russo, J. Appl. Phys. 101, 023115 (2007).

${ }^{77}$ A. Gojani, EPJ Web Conf. 45, 01124 (2013).

${ }^{78}$ M. H. Goetz, F. Baptista, J. P. Fischer, C. Messer, N. Suhm, and J. F. Bille, Proc. SPIE 2323, 218 (1995).

${ }^{79}$ G. M. Gale, G. Gallot, F. Hache, N. Lascoux, S. Bratos, and J.-C. Leicknam, Phys. Rev. Lett. 82, 1068 (1999).

${ }^{80}$ C. Porneala and D. A. Willis, J. Phys. D 42, 155503 (2009).

${ }^{81}$ L. I. Sedov, Similarity and Dimensional Methods in Mechanics (Academic Press, New York, NY, 1959). 